\title{
Oportunidades e Restrições para o Veículo Conectado: Foco em Sistemas de chamada de Emergência e Serviços
}

\begin{abstract}
Autores
Takahira, Ricardo Y. ${ }^{1}$, Tonello, Gisele ${ }^{2}$ e Luz, Marcio ${ }^{3}$

RTC2 ${ }^{1}$-Research\&Technology Consulting GMB-OnStar ${ }^{2}$ General Motors do Brasil Div. OnStar

Volvo do Brasil ${ }^{3-}$ Volvo Cars - Div. Wireless Car

E-mails: ricardo_takahira@hotmail.com, gisele.tonello@gm.com; márcio.luz@volvo.com

Co-participações : Luciano Alakija - Cisco (Jasper), Carlos Briselli - Renault (coordenador GT Conectividade Anfavea), - Fernando / David Borges - Ford, Henrique Miranda - BMW

\section{RESUMO}

Este trabalho discorre sobre a necessidade e oportunidade de ações focadas em telecomunicações e infraestrutura viária urbana e rodovias, para melhor introdução e segurança na adoção de tecnologias emergentes para o veículo conectado, integrado às tendências de eletrificação (hibrido e puro elétrico) e autonomia (veículo autônomo).

Com a Oferta de vários serviços pelas OEM's: SYNC, OnStar, BMW Connected Drive, Volvo Cars, uma das características é o sistema de chamada de emergência, presente em vários serviços ofertados na aquisição do veículo, uma das fragilidades na efetiva operação, no entanto é a cobertura Celular GSM, M2M principalmente nas estradas afastadas dos grandes centros urbanos.
\end{abstract}

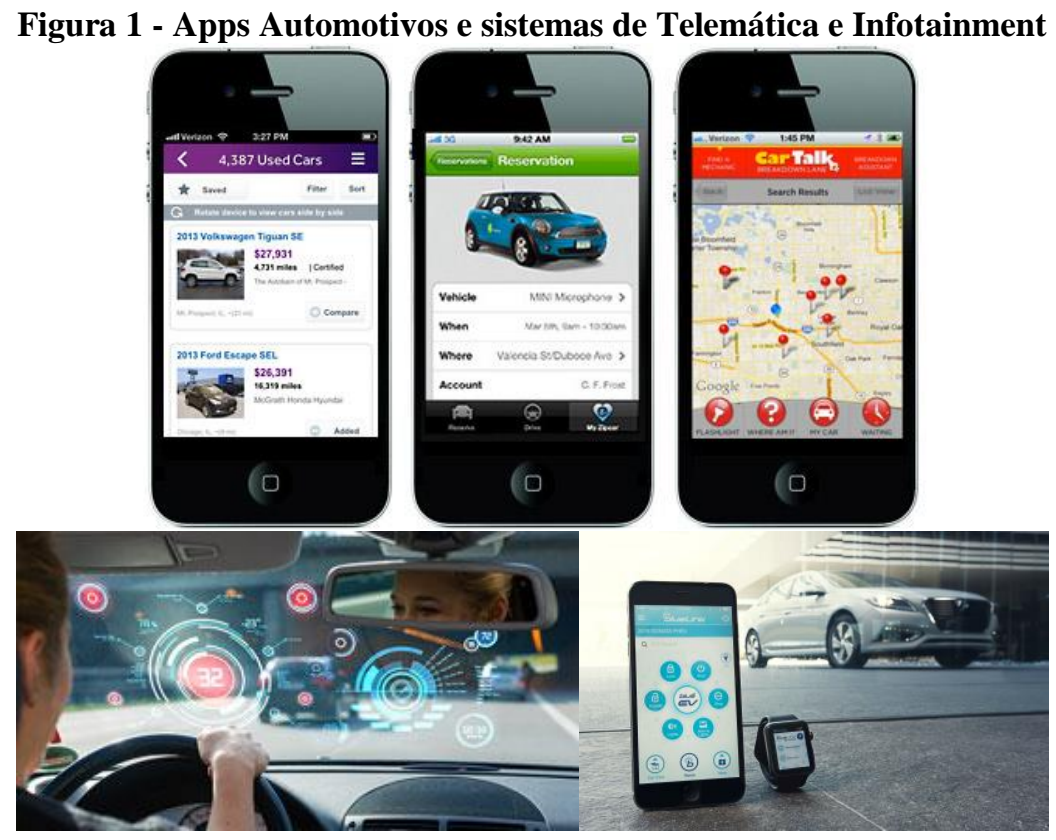




\section{INTRODUÇÃO}

Veículos Conectados são um dos desejos de consumo acompanhado das tecnologias para os Veículos Autônomos e Elétricos, este último mais ligado a exigências de emissões e eficiência energética. A tecnologia atual oriunda da Europa, Ásia ou Estados Unidos sofre com a cobertura de infraestrutura de comunicações no país que tem uma extensão territorial muito maior que o continente Europeu e regras de cobertura de sistemas de telecomunicações nas estradas não obrigatório. O que remete a um não reconhecimento da existência e operação do veículo Conectado e as soluções de serviço associadas. Muitos outros serviços poderiam ser explorados caso a cobertura nacional fosse mais abrangente, com benefícios aos próprios operadores das rodovias, concessionárias e autoridades de fiscalização como Policia Rodoviária Estadual e Federal que pudessem usar com confiança as comunicações para receber e enviar mensagens dos veículos e usuários das rodovias, gerando segurança, conforto e eficiência para os usuários aumentando o interesse de apressar a vinda de produtos e serviços conectados aos meios de transporte e malha viária do país. Como tudo os sistemas de transporte começam a ficar cada vez mais dependentes da infraestrutura das estradas, comunicação e tecnologias embarcadas e instaladas nas vias, rodovias e veículos.

Abstract: Connected Vehicles is one of the consumer desires accompanied by the technologies for the Autonomous and Electric Vehicles. The current technology coming from Europe or the United States suffers from the coverage of communications infrastructure in the country that has a much greater territorial extension than the European continent and rules of coverage of non-mandatory road telecommunication systems. This refers to a non-recognition of the existence and operation of the Connected vehicle. Many other services could be exploited if national coverage were more comprehensive on highways by operators, concessionaires and inspectors such as State and Federal Highway Police could use the communications to receive and send messages from vehicles and road users, generating safety, comfort and efficiency for the passengers increasing the interest, rushing the arriving of products and services connected to the transport modals and road net of the country. As all transportation systems become increasingly dependent on road infrastructure and communication more embedded technologies are installed in roads, highways and vehicles.

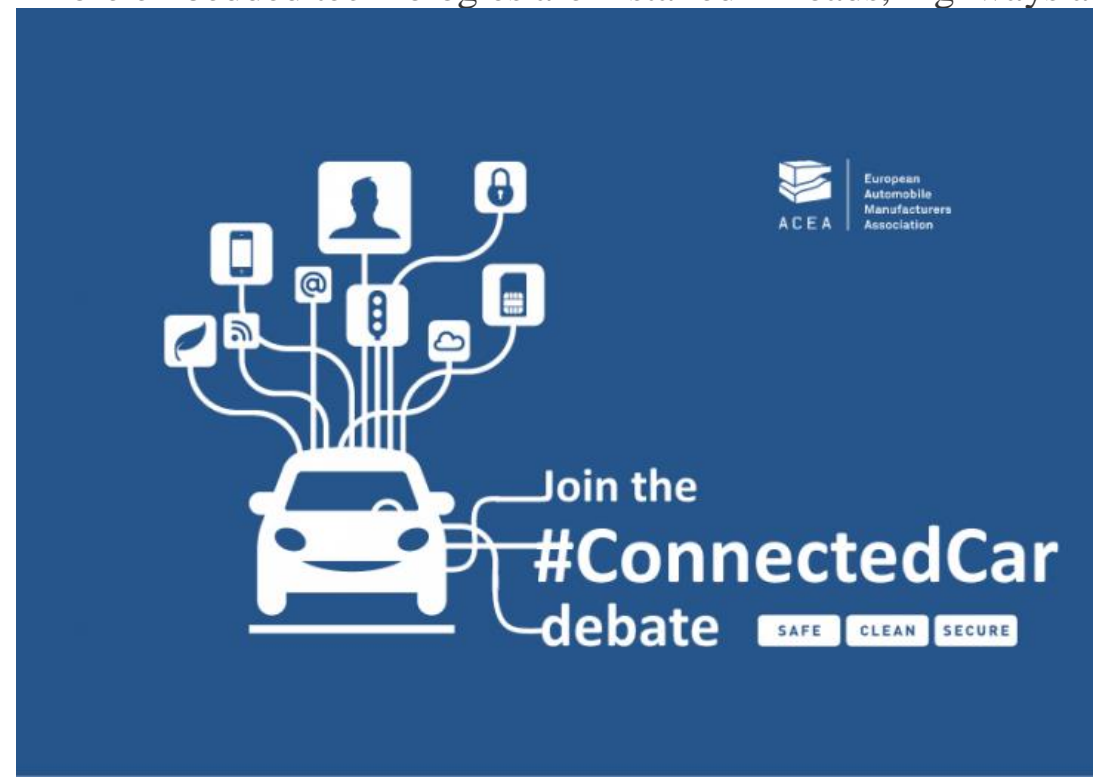

Figura 2 - Safe, Clean, Secure Connected Car 


\section{INFORMAÇÕES GERAIS}

Muito já se falou sobre veículos conectados V2X, onde V2I = Veículo com Infraestrutura,

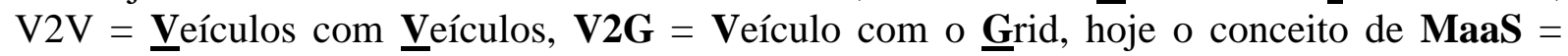
$\underline{\text { M}}$ obility $\underline{\boldsymbol{a}}$ s $\underline{\boldsymbol{a}} \underline{\text { Service }}$ vem sendo cada vez mais difundido, ou seja a troca de um modelo de posse de transporte individual para um uso do veículos compartilhados ou como serviço de transporte com maior uso de transporte coletivo, transporte compartilhado em modelos de uso ao invés de posse priorizando a integração de modais e serviços: Ônibus, Trens, VLTs, Metro, Taxi, Uber, Veículos compartilhados, bicicletas, trechos à pé com áreas e corredores integrados priorizando a integração de modais e respeitando a hierarquia de mais vulneráveis no transito em temos de proteção.

É evidente que a maioria destas soluções são mais utilizáveis em grandes centros urbanos ao invés de cidades mais afastadas e áreas rurais e é aí que incorremos em um erro gravíssimo, a falta de cobertura de tecnologias de telecomunicação para permitir cada vez mais o uso de aplicativos móveis em harmonia com o conteúdo de eletrônica embarcada dos novos carros no mercado:
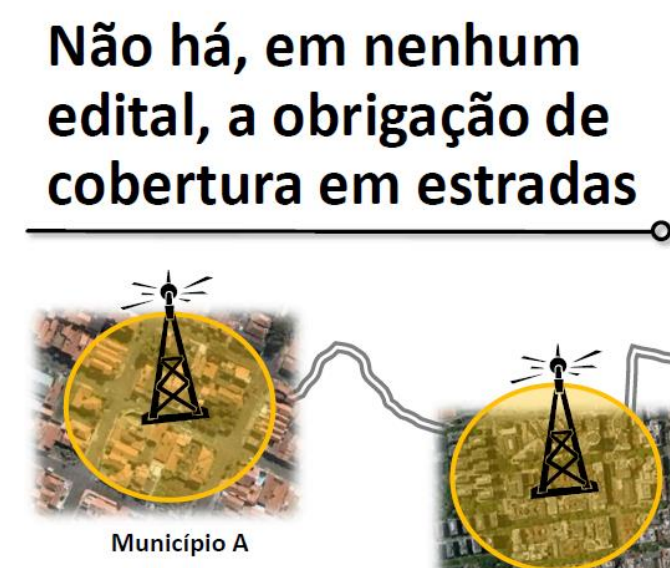

Município A

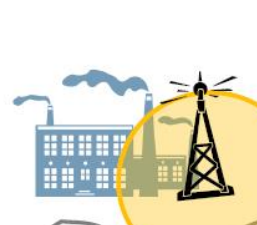

Área de cobertura viável economicamente

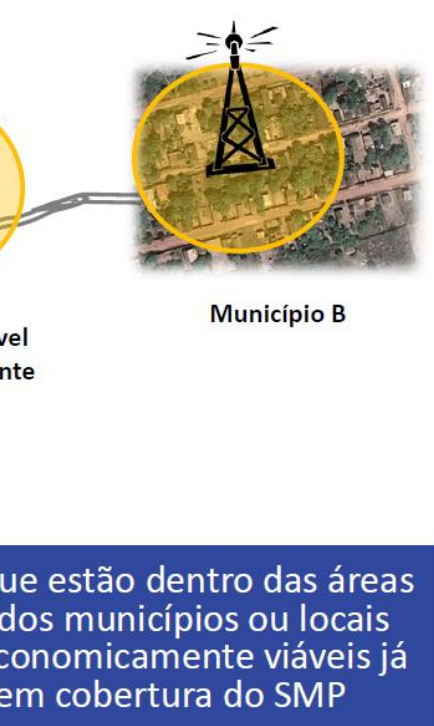

Figura 3 - Fonte Sinditelebrasil - Cobertura nas Estradas não obrigatória

estradas que estão dentro das áreas

urbanas dos municípios ou locais

que são economicamente viáveis já possuem cobertura do SMP

O fato da Anatel ainda não regular a obrigatoriedade de cobertura nas estradas tem razão de ser: $\mathrm{O}$ foco sempre foram os habitantes das grandes metrópoles priorizando o investimento de infraestrutura $\mathrm{ERB}=$ Esstação $\underline{\text { Rádio }}$ Base para o maior número de habitantes e usuários, porem após alguns anos de implementação das tecnologias 3G e 4G, agora na eminencia de regras para o $5 \mathrm{G}$, os equipamentos $3 \mathrm{G}$ poderiam ter uma destinação de cobertura nacional, iniciando pelas estradas sob concessão e caso as operadoras ainda não tenham interesse em investir visto o cálculo óbvio de investimento x retorno em usuários, as concessionárias poderiam se associar operando como MVNO, substituindo a obrigação de postos de SOS (telefones distribuídos a cada $1 \mathrm{Km}$ ) na zona de concessão por cobertura Celular ou equivalente de comunicação. A questão da gratuidade ainda pode ser mantida por serviços 0800 . 


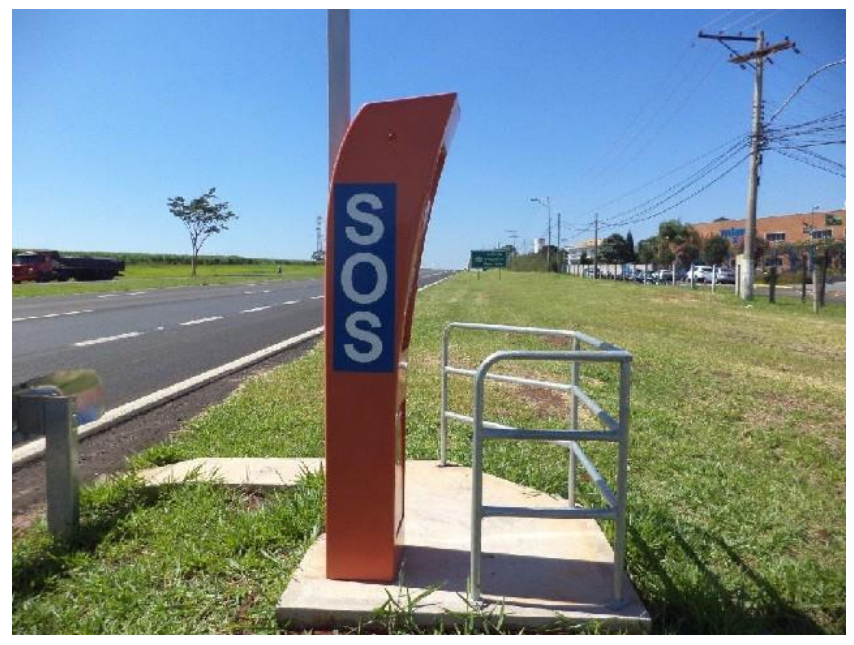

\section{Da infraestrutura nas estradas:}

1.1. Os novos editais de concessão das estradas devem propor outros mecanismos de infraestrutura de telecomunicações para as concessionárias como WiFi.

1.2. Alinhar as licitações com as regulamentações é difícil, mas estas oportunidades coordenadas é que fazem com que seja possível disseminar as tecnologias em tempos e prazos hábeis com a segurança de regulamentação especifica.

1.2.1. Todas as consultorias especializadas apontam o carro conectado como tendência tecnológica e área de maior crescimento dentro do segmento.

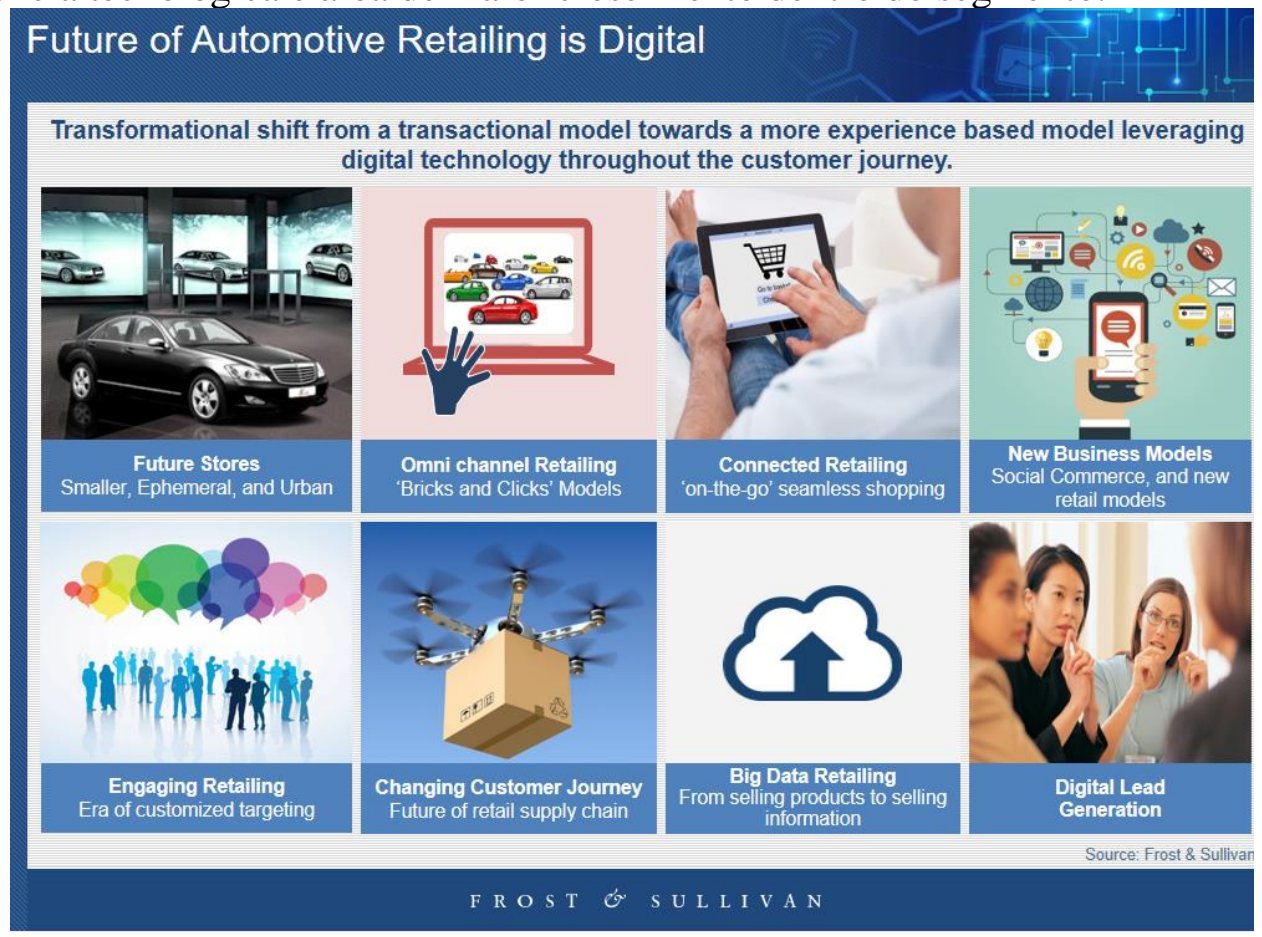

Figura 4 - Megatendência Carro Conectado - Fonte: Frost \& Sullivan GIL 2017

1.2.2. Muitas, no entanto se baseiam nas informações e lançamentos e crescimento internacional, faltam levantamentos e mapeamento local especifico. 


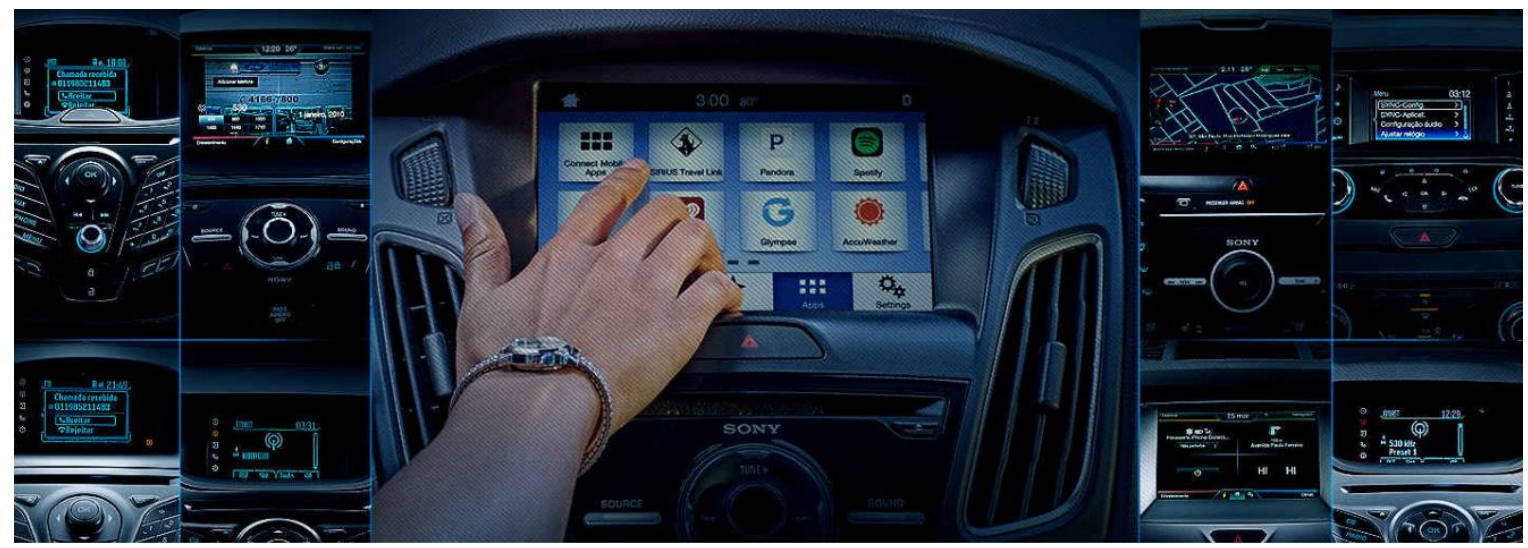

Figura 5 - Fonte SYNC Ford - A Telemática e Infotainment podem evoluir muito com infraestrutura de Telecom nacional

\section{Da oportunidade de cobertura como MVNO - Mobile Virtual Network Operator}

2.1. O Brasil é um país vasto de grandes extensões continentais e diferenças Econômicas e culturais, cabendo no mesmo vários países da Europa:

\section{Defining the Problem: Connectivity Infra in Brazil}
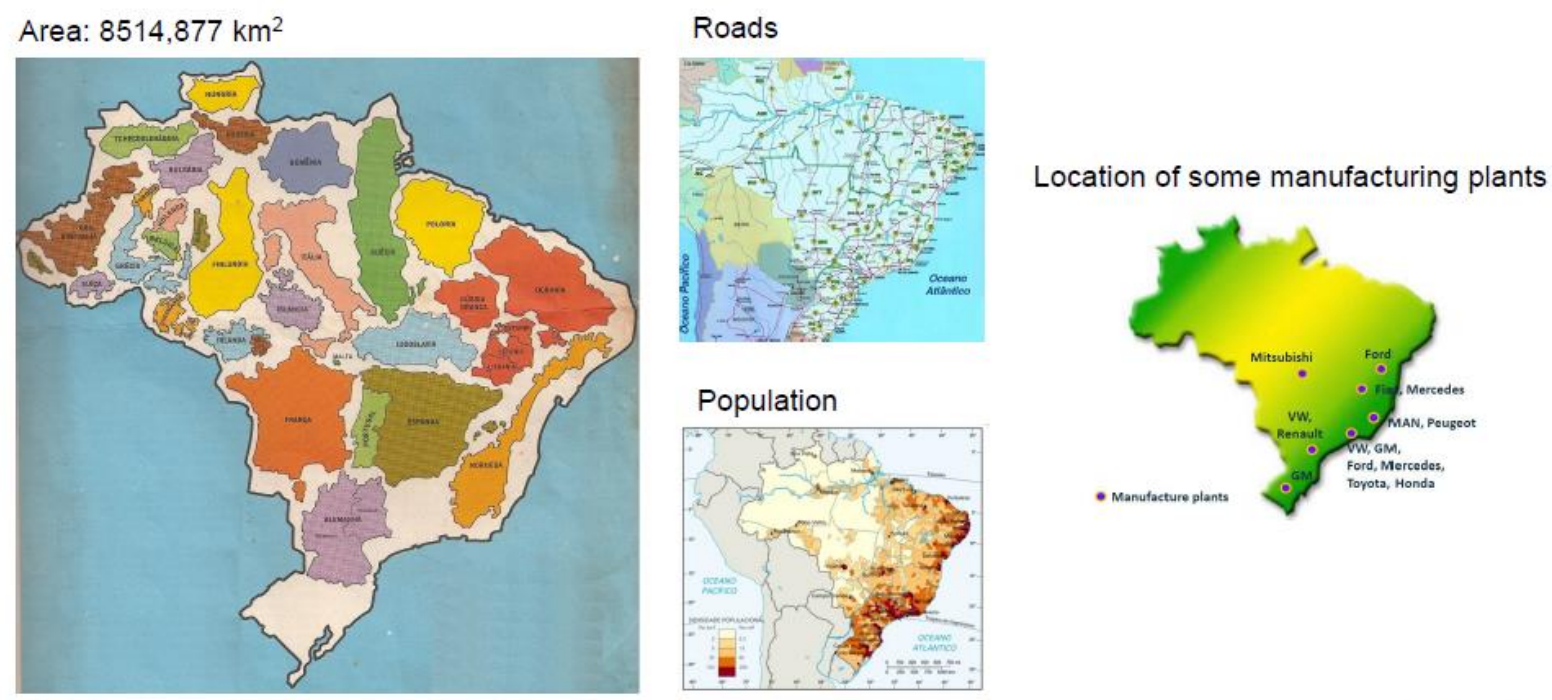

25 connoentla

Figura 6 - Fonte: Cisco Jasper - Sinditelebrasil

2.2. Pelo modelo de negócio em escala algumas operadoras poderão preferir se concentrar nos grandes centros urbanos, atualmente é possível operar como virtual no serviço de telecomunicações, assim concessionárias que já operam os serviços e pedágios das rodovias poderiam trocar a obrigatoriedade da instalação e manutenção dos telefones fixos SOS por estações rádio base, que além dos usuários podem trazer grandes benefícios aos servidores das estradas e policias rodoviárias estatuais e Federais, mas o melhor ganho é sim o GOLDEN MINUTE, traduzindo: cada minuto ganho no socorro pode significar menos sequelas nas vítimas sempre. 


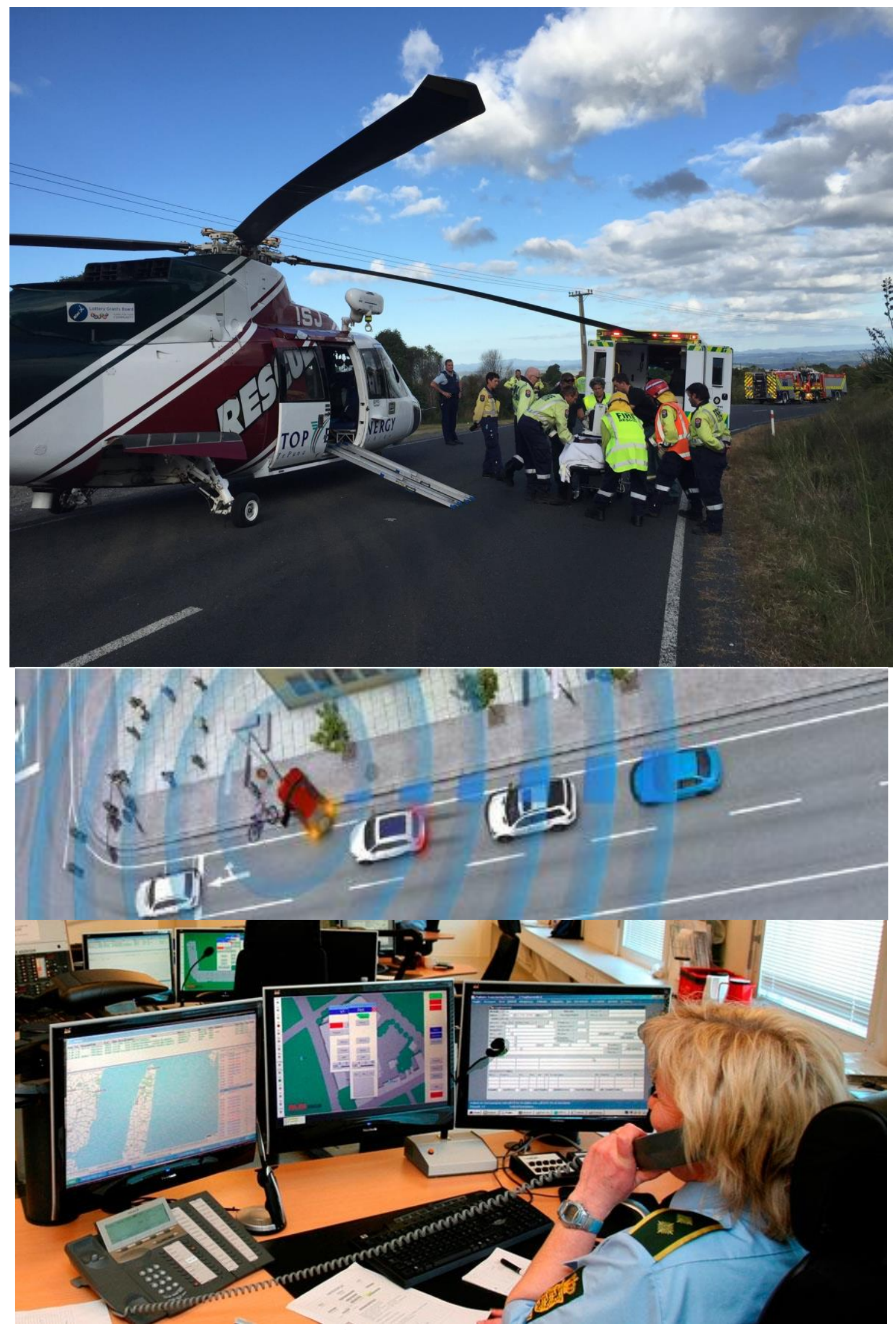

Figura 7 - Sistemas de chamada em emergencia 
2.3. Outro ganho indireto está relacionado ao Agronegócio e sistemas de rastreamento e telemetria do transporte comercial, ajudando a conectividade agrícola do campo à logística de escoamento de grãos e produtos, em um segmento muito representativo para economia do país.

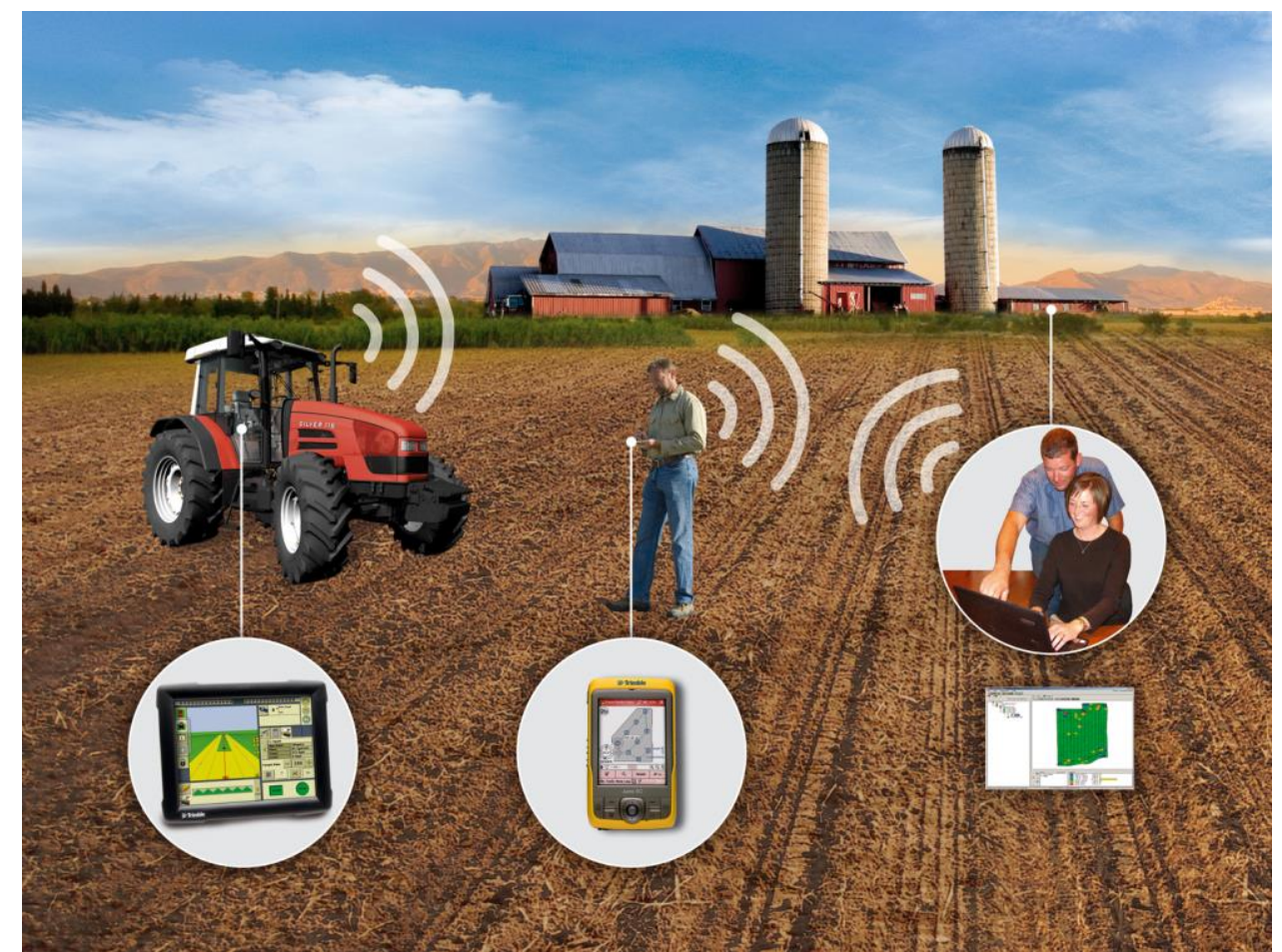

Figura 8 - IoT no agronegócio ou cobertura GSM nas estradas pode beneficiar muito o agronegócio

2.4. Porem isso não vai resolver o problema como um todo, mas pode amenizar muito a falta de infraestrutura não só para o veículo conectado, mas dar um passo significativo em outra tendência muito comentada: a internet das coisas, mais especificamente neste nosso exemplo e segmento IoTT - Internet of Things on Transportation.

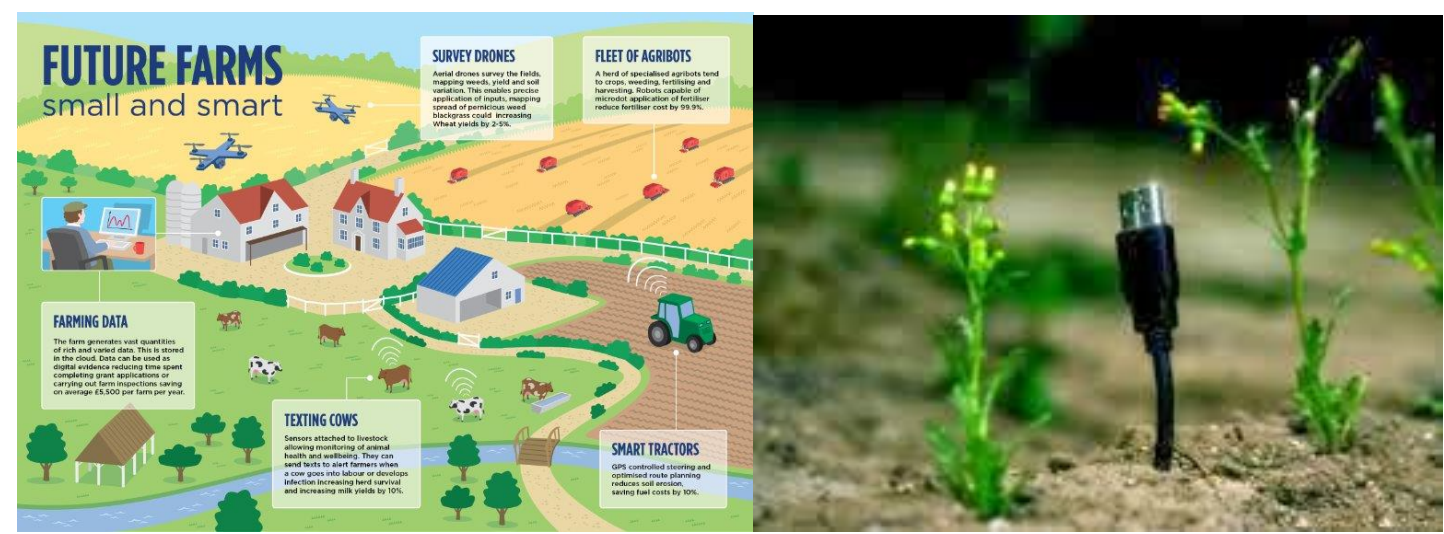

Figura 9 - Ecossistema com conectividade em Agronegócio

3. Atuais serviços em comercialização no pais já contemplam a chamada de emergência autônoma nos veículos apenas diferem na forma de acionamento, característica da operação e treinamento dos seus próprios call centers 
3.1. Mais serviços agregados poderiam ser implementados caso a rede fosse de maior abrangência

3.2. Para sistemas baseados em SIM CARD e Smartphones a cobertura celular é mandatória, quando sem crédito a ligação em emergência 19X não é possível fora da área da operadora de contrato de origem.

3.3. A classificação fora de serviços puro $\mathrm{M} 2 \mathrm{M}$, para as atuais soluções embarcados também não desonera o sistema do onto de vista de taxas de ativação e manutenção. 
Serviços de Emergência presente nos veículos conectados no mercado Nacional:

\begin{tabular}{|c|c|c|c|c|c|}
\hline Montadora: & Produto e/ou Serviço: & $\begin{array}{l}\text { Chamada de } \\
\text { Emergência: }\end{array}$ & Modo: & $\begin{array}{l}\text { B - } \\
\text { Call? }\end{array}$ & Site: \\
\hline FORD & FORD SYNC & SIM & $\begin{array}{l}\text { Celular } \\
\text { Pareado BT }\end{array}$ & SIM & https://www.ford.com.br/servico-ao-cliente/sync/ \\
\hline GM & OnStar & SIM & $\begin{array}{l}\text { M2M- } \\
\text { SimCard }\end{array}$ & SIM & http://www.chevrolet.com.br/onstar.html \\
\hline VOLVO & VOLVO On CALL & SIM & $\begin{array}{l}\text { M2M- } \\
\text { SimCard }\end{array}$ & SIM & $\begin{array}{l}\text { http://www.volvocars.com/br/proprietario/informacao-proprietario/volvo- } \\
\text { on-call }\end{array}$ \\
\hline \multirow[t]{2}{*}{ BMW } & $\begin{array}{l}\text { BMW Connected } \\
\text { Drive }\end{array}$ & SIM & $\begin{array}{l}\mathrm{M} 2 \mathrm{M}- \\
\text { SimCard }\end{array}$ & SIM & https://www.bmw-connecteddrive.com.br/app/br/index.html\#/portal \\
\hline & & & & & $\begin{array}{l}\text { https://www.bmw-connecteddrive.com/country-select/country- } \\
\text { selection.html }\end{array}$ \\
\hline
\end{tabular}

Todas as montadoras OEM foram consultadas para preenchimento da Tabela acima durante as plenárias do CT EE AEA - Comitê Técnico de Eletroeletrônica Automotiva da Associação Brasileira de Engenharia Automotiva.

Os serviços diferem na sua oferta e podem ser consultados a partir das páginas disponíveis na Internet.

Fica claro o foco apenas na chamada de emergência, na proposta de recomendação de melhorias e possíveis mudanças regulamentárias para os atores do sistema: Operadores de Telefonia Celular e Concessionárias de serviços nas rodovias, reguladas especificamente pelas agencias Federais, Estaduais e Municipais. 
Não é em nenhum momento interferir nas estratégias individuais das OEMs, sistemistas, provedores de serviço, operadoras de telefonia

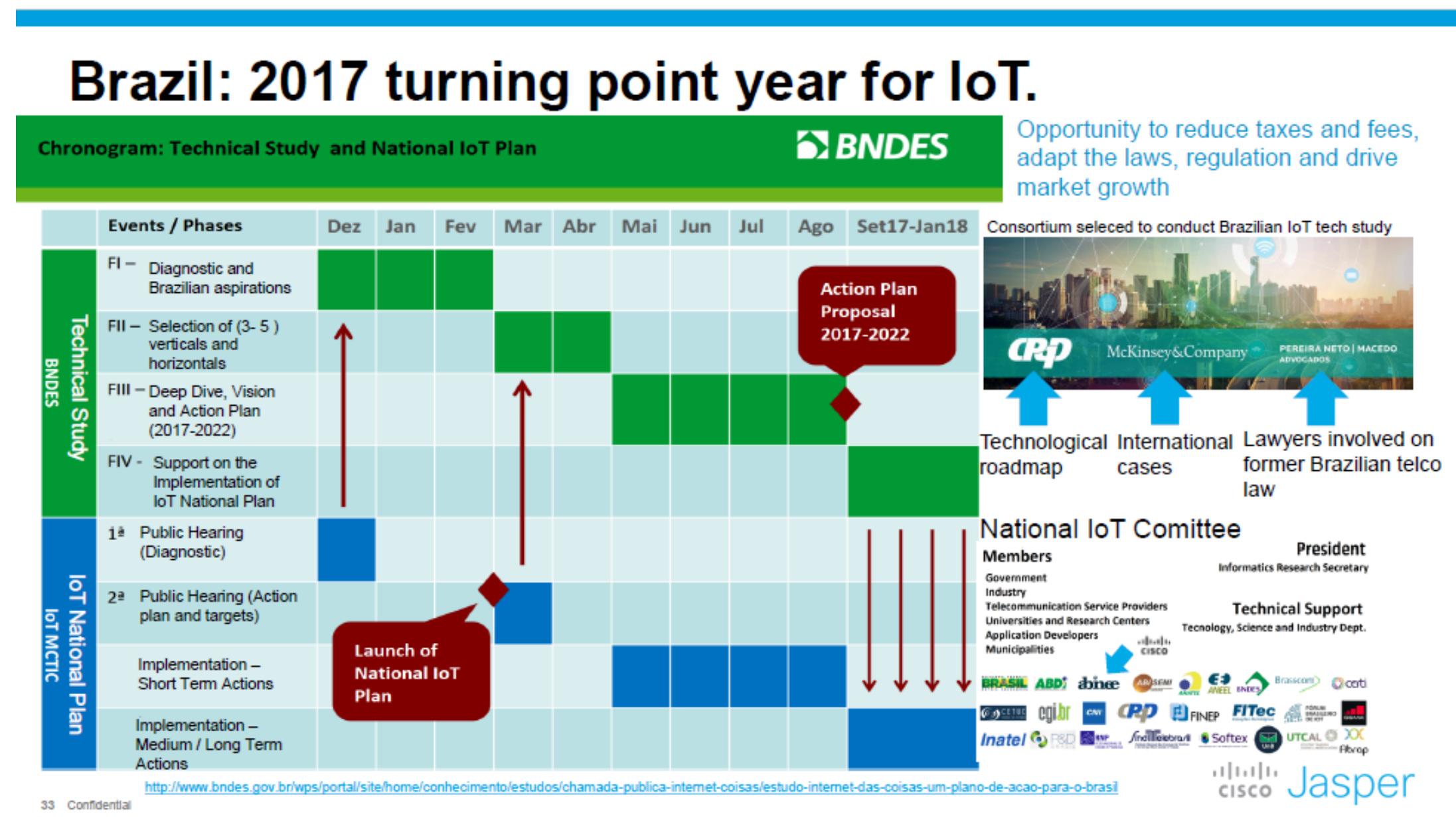

celular nem tampouco nas agências regulatórias governamentais, mas sim promover o diálogo, conhecimento e principalmente a integração entre operadores, provedores de serviço alinhando a oferta de conteúdo embarcado para melhor performance a maior uso dos serviços atuais e por vir. 


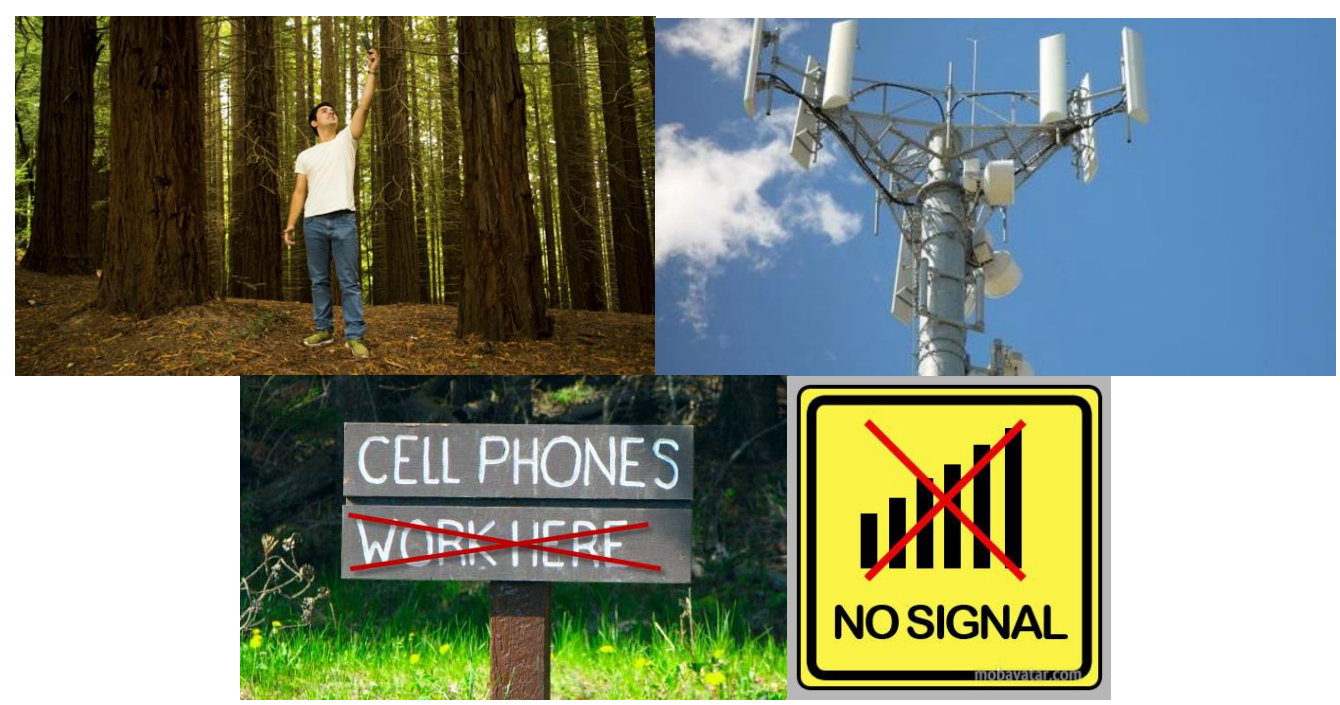

\section{CONCLUSÃO}

Visto a análise do cenário automotivo e sua relação com outras de aplicação e sua dependência de áreas externas, e pela complexidade de sistemas do veículo conectado, surge a urgente necessidade de uma ação articulada com outros atores não tradicionalmente ligados à indústria automotiva e os serviços que agora se apresentam. Se o serviço existe e é ofertado no exterior, neste momento de reavaliação de política industrial do setor, os sistemas eletrônicos embarcados, soluções de eficiência energética (eletrificação) e Emissões (Sustentabilidade Ambiental) exigem uma discussão e integração de diálogo com outros setores fora da indústria automotiva como agências de transportes e estradas, telecomunicações, TI e segurança da Informação principalmente quando se iniciam estudos para IoT e leilões 5G. É também o momento oportuno de estudar, incorporar e colocar metas e regras no programa Rota 2030 com revisões periódicas constantes, pois a tecnologia integrada nestes produtos evolui muito mais rapidamente do que novas regras possam ser definidas e garantidas. 
Para esclarecimentos adicionais, contatar:

AEA - Associação Brasileira de Engenharia Automotiva

Rua Salvador Correia, 80 - Aclimação

São Paulo - SP - 04109-070

Tel.: (011) 5908-4040

E-Mail: eventos@aea.org.br

\section{REFERÊNCIAS}

http://sinditelebrasil.org.br/ Acessado em 02 de junho 2017

http://sinditelebrasil.org.br/posicionamentos/contribuicoes-em-consultas-publicas Acessado em 02 de junho 2017

https://www.ford.com.br/servico-ao-cliente/sync/Acessado em 02 de junho 2017

http://www.chevrolet.com.br/onstar.html Acessado em 02 de junho 2017

http://www.volvocars.com/br/proprietario/informacao-proprietario/volvo-on-call Acessado em 02 de junho 2017

https://www.bmw-connecteddrive.com.br/app/br/index.html\#/portal Acessado em 02 de junho 2017

https://www.bmw-connecteddrive.com/country-select/country-selection.html Acessado em 02 de junho 2017

http://abcr.org.br/ Acessado em 02 de junho 2017

http://www.grupoccr.com.br/ Acessado em 02 de junho 2017

Acessados em junho 2017:

https://www.gsma.com/iot/wp-

content/uploads/2013/02/GSMA_mAutomotive_TechnologyRoadmap_v2.pdf

https://www.gsma.com/iot/wp-content/uploads/2012/03/gsma2025everycarconnected.pdf

http://www.acea.be/industry-topics/tag/category/connected-and-automated-driving

http://www.acea.be/events/event/connected-cars-europe-2016

http://www.automotiveworld.com/news-releases/acea-connected-automated-driving-eata-

presents-deployment-roadmap-submits-proposal-eu-project/

http://www.acea.be/press-releases/article/connected-and-automated-driving-eata-presentsdeployment-roadmap-submits-pr

http://www.acea.be/press-releases/article/ministers-eu-policymakers-and-auto-industry-pushfor-connected-and-automate

https://assets.kpmg.com/content/dam/kpmg/xx/pdf/2017/01/global-automotive-executivesurvey-2017.pdf

https://www.smmt.co.uk/industry-topics/technology-innovation/connected-autonomous-

vehicles/

http://convergecom.com.br/teletime/20/09/2016/bndes-e-mctic-definem-consorcio-formado-

pela-mckinsey-e-cpqd-para-desenvolver-plano-de-iot/

http://www.docs.ndsr.org/IoT_MaximilianoMartinhao.pdf

http://www.rfdtv.com/story/35132817/connected-car-m2m-2017-global-market-expected-togrow-at-cagr-2752-and-forecast-to-2021

http://www.mcti.gov.br/noticia/-/asset_publisher/epbV0pr6eIS0/content/mctic-anuncia-

diretrizes-do-plano-nacional-de-internet-das-coisas-nos-proximos-dias 
http://www.abinee.org.br/informac/arquivos/aiot.pdf

http://www.eventos.momentoeditorial.com.br/wp-content/uploads/2017/03/THALES-

MARCAL.pdf

http://convergenciadigital.com.br/cgi/cgilua.exe/sys/start.htm?UserActiveTemplate=site\&info

$\underline{\mathrm{id}=43703 \& \text { post } \% 252525255 \mathrm{Fdata}=\& \mathrm{sid}=17 \# . \text { WTSUD4WcG00 }}$ 